Voix et Images

volxetimages

\title{
Théâtre au féminin : Encore 5 minutes et Les Fées ont soif
}

\section{André Smith}

Volume 7, numéro 2, hiver 1982

Michel Tremblay

URI : https://id.erudit.org/iderudit/200325ar

DOI : https://doi.org/10.7202/200325ar

Aller au sommaire du numéro

\section{Éditeur(s)}

Les Presses de l'Université du Québec

\section{ISSN}

0318-9201 (imprimé)

1705-933X (numérique)

Découvrir la revue

\section{Citer cet article}

Smith, A. (1982). Théâtre au féminin : Encore 5 minutes et Les Fées ont soif. Voix et Images, 7(2), 351-365. https://doi.org/10.7202/200325ar d'utilisation que vous pouvez consulter en ligne.

https://apropos.erudit.org/fr/usagers/politique-dutilisation/ 


\title{
Théâtre au féminin : Encore 5 minutes et Les Fées ont soif
}

\author{
par André Smith
}

Encore 5 minutes de Françoise Loranger et Les Fées ont soif de Denise Boucher furent deux pièces à succès. Toutes les deux traitent le même thème : l'insatisfaction féminine. Elles s'achèvent également d'une manière identique en affirmant la libération de leurs héroïnes.

Cette thématique (au sens large) et ce dénouement communs n'excluent pas que ces deux cuvres se distinguent d'une manière assez radicale.

D'abord, leurs points de vue diffèrent. Ecrite pour la radio en 1966, reprise pour la scène et la télévision en 1967, la pièce de Loranger est centrée autour d'un personnage. Elle met en scène une femme dont on approfondit la psychologie et les motivations. Elle est originale en ce qu'elle aborde la question de l'œdipe à partir de la mère et non du fils comme c'est le cas le plus souvent. II convient aussi de signaler qu'elle accorde une place importante aux relations mère-fille, ce qui n'est pas si fréquent dans notre littérature.

Certes, on peut faire une lecture féministe d'Encore 5 minutes, mais il reste que cette pièce a été écrite dans le dessein de représenter un cas individuel duquel l'auteur ne tire aucune leçon politique. Comme l'indique Jean-Pierre Crête, Françoise Loranger " met en scène une femme de cinquante ans qui remet en question toutes les idées reçues, constate qu'elle n'a jamais vécu par elle-même et décide de faire table rase afin de recommencer à neuf." ' Cette héroïne, Gertrude, livre un combat strictement personnel, vide de toute conscience militante, nullement ancré dans une démarche collective.

Les fées ont soif, au contraire, servent ouvertement la cause féministe. Cette pièce s'est même retrouvée au cœur d'une polémique qui est à l'origine de son succès. Cette approche politique est intéressante puisqu'elle permet d'observer les rapports complexes qui existent entre le fait littéraire et le projet idéologique. 
Dans cet article, nous examinerons ce qui distingue la démarche individualiste, "bourgeoise", de Loranger de celle, engagée, militante de Boucher. Notre comparaison portera sur les thèmes suivants: l'insatisfaction féminine, la maternité, la sexualité, le recours idéaliste et la libération des femmes.

\section{L'insatisfaction féminine}

L'insatisfaction féminine est donc le thème commun des deux œuvres étudiées. Comment ce thème évolue-t-il d'une pièce à l'autre?

Dans Les fées, la frustration de la femme est exprimée par des archétypes: la Vierge, l'épouse et mère, la putain. Au contraire, dans Encore 5 minutes, cette même frustration est incarnée dans un personnage : Gertrude. D'un côté donc, une femme conceptualisée, représentée dans ses rôles. De l'autre, une femme réduite à sa vie intérieure, soumise à une crise existentielle...

Dans Les Fées, l'aliénation féminine est posée comme un postulat et la libération de la femme comme une nécessité. D'une part, il y a le Mal; d'autre part, la solution au Mal. Nous sommes en présence d'un point de vue militant que l'œuvre a pour fonction d'illustrer. Cela est visible dès le début de la pièce ${ }^{2}$ alors que les trois femmes expriment fortement l'insatisfaction que leur cause leur rôle. Deux remarques s'imposent ici.

D'abord, si le point de vue de Denise Boucher est vraisemblable, il n'est pas universel. II ne va pas nécessairement de soi. Une prostituée, une mère de famille ne sont pas automatiquement accablées, dépourvues d'identité, désespérément frustrées. À cet égard, la vision de Denise Boucher m'apparaît davantage morale que littéraire. Ses femmes sont des concepts qui permettent d'enclencher le discours féministe. Car il faut que la statue, Marie et Madeleine solent malheureuses afin que leur lutte devienne nécessaire.

Par ailleurs, ce parti-pris politique n'exclut pas que la pièce conserve, le plus souvent, son efficacité dramatique. Elle ne nous semble pas composée, comme l'écrit non sans méchanceté Jean-René Éthier, « de signes sans densité, contradictoires et peu adaptés à l'expression du thêâtre lui-même. „33 Sans doute, l'écriture de Denise Boucher est-elle parfois complaisante, par exemple lorsqu'elle fait dire à la statue: «ils m'ont enfermée au cœur mème de la chair de la pomme»4; ou encore: "je suis le léchage de la dénégation $" 5$. Dans l'ensemble cependant, le message est clair : il consiste en une dénonciation généralisée de la condition féminine.

D'un point de vue dramatique, cette dénonciation se déroule selon un processus assez simple. D'une part, Marie et Madeleine exposent leurs griefs. D'autre part, la statue commente leur plainte : elle exerce ainsi une fonction critique dans la pièce. 
Par exemple, lorsque Madeleine se plaint de jeter « les spermatozoïdes par les fenêtres" 6 et de vivre une sexualité stérile, qui prive le pays d'enfants, la statue ponctue avec humour cette nostalgie de maternité: "Comme je disais à Fatima : pauvre Canada " 6 .

Cette manière, chez Denise Boucher, de faire suivre l'expression d'une frustration par une réflexion critique apparaît comme l'un des procédés les plus habiles de l'œuvre. Tantôt ironique et marquée par un anticléricalisme gentiment désuet, comme cette allusion à Fatima, tantôt plus actuelle, plus authentiquement féministe, cette réflexion critique procure un effet de distanciation qui donne à la pièce toute son efficacité politique.

Madeleine regrette donc de se prostituer. Elle rêve d'être mère, d'être aimée. Elle voudrait bien “se fair' appeler maman " ${ }^{7}$ et «croire à l'amour " ${ }^{8}$. Le mariage conserve même quelque séduction chez elle puisqu'elle souhaite "un bon mari comme amant» 7 . Un tel conformisme ne manque évidemment pas d'être sanctionné par l'auteur qui utilise à cette fin deux procédés.

D'abord, le rêve de Madeleine est ponctué d'une chanson ironique, "Un jour mon prince viendra», placée dans la bouche de la statue qui exerce de nouveau ainsi sa fonction corrosive, dénonciatrice.

Ensuite, d'une manière plus structurelle, plus définitive, Marie, l'épouse et la mère de la pièce, fait ressortir la vanité du projet de Madeleine. Car Marie est malheureuse. Elle n'a plus aucun désir sinon celui, inhérent à son rôle de ménagère, d'acquérir de «nouveaux savons qui rendent le linge encore plus blanc, plus propre " 8 . Elle se plaint aussi de son mari qui l'a tout juste effleurée comme «le vent qui ment»?.

Prostituée ou épouse, la femme de Denise Boucher est donc fondamentalement insatisfaite. Nul avenir ne se profile devant elle, car la société mâle - ici le client, là le mari - compose "un ordre qui mange " ${ }^{10}$ son identité.

Reste le personnage de la Vierge, certainement le plus original des Fées ont soif. Chez Denise Boucher, la Vierge, c'est un système de valeurs qu'il convient de dénoncer, c'est une image qu'il faut briser. En s'attaquant au mythe de la Vierge, tellement important dans notre civilisation, l'auteur accomplit avant tout un acte de subversion idéologique. A une Vierge traditionnelle, asexuée, proposée comme modèle féminin depuis l'avènement du christianisme, Denise Boucher substitue une Vierge révoltée, corrosive, critique, qui se fait la complice et l'écho de l'épouse et de la prostituée qui souhaitent sortir de leur condition. Voulant cesser d'être «le symbole pourri de l'abnégation pourrie ${ }^{9}$, la Vierge décide de "descendre de l'arbre ${ }^{7}$ pour demander : “Qui me déviergera?»"10.

En un mot, avant le combat féministe, la femme est fragmentée. Tour à tour putain, mère de famille, caution idéalisée et évanescente de l'ordre chrétien, c'est-à-dire masculin, elle ne maitrise ni ses plaisirs ni ses 
maternités ni ses valeurs. Le projet des Fées ont soif consiste à abolir le mythe de la Vierge et à réunir en une seule personne, maîtresse d'elle-même, la maman et la putain.

L'insatisfaction féminine est donc traitée avec optimisme dans les Fées ont soif. L'aliénation de la femme est exposée, ses causes sont disséquées et des solutions sont entrevues. Cet optimisme reflète bien sûr davantage les convictions et les parti-pris de l'auteur que la réalite. On peut déplorer, par exemple, que la pièce n'aborde guère la question de l'argent. Pourtant, l'un des principaux bastions du pouvoir mâle dans notre société, c'est bien le pouvoir de dépenser. Pour le meilleur et pour le pire, ce qui définit le citoyen moderne, c'est sa qualité de consommateur. À cet égard, les Fées ont soif nous parlent peut-être trop de la Vierge et pas assez de la sujétion économique dans laquelle vivent la plupart des femmes de tous les milieux sociaux.

Le malheur de Gertrude relève donc du drame individuel. Nulle idéologie, aucune forme de féminisme, qui puisse calmer ce type d'angoisse. A cet égard, Gertrude présente peu d'intérêt politique.

Mais revenons au decor d'Encore 5 minutes. Le seul moment de paix dont profite l'héroïne, la seule occasion où elle n'entend avec son mari, c'est lorsqu'elle accepte de s'installer dans un arrangement traditionnel ${ }^{11}$. Mais cela ne dure pas. Non seulement, Gertrude ne veut pas s'asseoir "sur n'importe quoi "12, mais encore souhaite-t-elle vivre dans un décor de jeunesse qui "jaillisse du plus creux de ses entrailles, du plus profond " 13 d'elle-même. Il y a donc chez elle un puissant désir de s'affirmer en s'appropriant la chambre de sa fille. Ce désir confine d'ailleurs à l'obsession, car c'est depuis trois "semaines que Gertrude vit du matin au soir dans cette pièce" ${ }^{13}$, incapable de donner une forme à son fantasme comme si une pudeur refoulée, une interdiction secrète l'empêchait de s'emparer du décor de Geneviève.

Gertrude n'arrivera d'ailleurs à aucun moment à dominer cet espace qu'elle désire pourtant avec violence. Au début du deuxième acte, elle est "toujours là dans cette pièce où les choses maintenant s'accumulent" 14 . Elle vit dans une énorme confusion qui accable son entourage. Son échec est d'ailleurs symbolisé par une fissure qui lézarde le mur et dont on trouve la trace dans de nombreux passages. ${ }^{15}$ Finalement, faute de pouvoir aménager la chambre de sa fille à son profit, Gertrude finit par glisser dans une sorte de folie. Elle imagine sa pièce toute blanche et elle renonce à l'habiter.

Comme avec son décor, Gertrude entretient de très mauvais rapports avec son mari qui affirme à leur sujet: "ll y a comme ça des couples privilégiés pour qui le mariage est un combat à mort" ${ }^{16}$. Henri affirme d'ailleurs avoir épousé Gertrude par imbécillité. ${ }^{17}$ 
C'est donc sans surprise que l'on relève les différents aspects du conflit qui déchire le couple. À cet égard, Encore 5 minutes fait penser au théâtre bourgeois de Marcel Dubé. Ces couples malheureux, qu'on a tendance à trouver aujourd'hui ennuyeux et dépourvus d'intérêt, étaient au contraire pris au sérieux durant les années soixante.

Par exemple, le passage dans lequel Henri n'ose gifler sa femme qui le défie de le faire aurait sans doute intérêt, maintenant, à être mis en scène d'une manière comique, qui insiste sur le côté grotesque et dérisoire de l'incident. Il en va de même des scènes dans lesquelles Gertrude se plaint d'être trompée, son infortune ne pouvant plus guère que susciter un sourire amusé du public.

Notre héroïne vit cependant son malheur avec sérieux. Ainsi, lorsque son mari veut croire à la restauration de l'ordre familial, elle le ridiculise en le traitant de «Pauvre fou» 18 . Elle le réduit aussi systématiquement à un rôle pratique en éructant à son endroit : "Une chaise! C'est tout ce que je te demande." 19 Au-delà, elle souhaite le chasser une fois pour toutes : "Mon cher Henri, quand cette pièce sera terminée, vous n'y remettrez plus jamais les pieds » 20 . On touche ici à l'ambiguiité qui fait tout l'intérêt d'Encore 5 minutes. Car ce n'est pas seulement Henri que Gertrude désire liquider, mais tout le réel, y compris, à la limite, elle-même. Elle veut disparaître pour renaître dans la personne de sa fille. En ce sens, Henri ne manque ni d'à propos ni de cruauté lorsqu'il affirme : "Une mère et une fille, Gertrude, ça ne fait pas un, ça fait deux! II faut que tu le comprennes. "16

Mais pourquoi Gertrude désire-t-elle s'identifier à Geneviève? Tout simplement parce que cette dernière a fait une fugue avec son amant et que sa mère s'efforce de revivre, à travers les amours de Geneviève, la grande aventure de sa jeunesse. Bien sûr, la distance aidant, Gertrude magnifie son passé amoureux, ce qui permet a Henri, encore une fois, d'exprimer avec plaisir une vérité déplaisante pour sa femme. Il lui dit en effet que le "grand imbécile " 21 qu'elle idéalise ne l'a jamais aimée : "Tu n'as même pas eu le choix de t'enfuir avec lui, parce qu'il n'en a jamais été question!»22

Epouse malheureuse, Gertrude éprouve une insatisfaction purement existentielle. II n'y a pas chez elle l'embryon d'une conscience féministe. Elle est engluée dans son narcissisme, attentive à ses seuls sentiments et tente, pour échapper à son spleen, de se construire un théatre intime dans lequel tous les autres, et singulièrement sa fille et son fils, ne sont que des pantins. Alors que Marie, dans les Fées ont soif, s'efforce d'échapper à un mauvais mariage en s'unissant, pour ainsi dire politiquement, à Madeleine, l'héroïne d'Encore 5 minutes, plus traditionnelle, plus égoïste, cherche à se libérer intérieurement en utilisant les siens. Cela ressort nettement quand on étudie le thème de la maternité dans les deux pièces. 


\section{La maternité}

Comme on l'a vu, Gertrude s'efforce d'ordonner le monde en fonction de ses fantasmes. Cela est particulièrement clair dans ses rapports avec ses enfants. Elle fait ainsi vivre à sa fille sa propre vie en lui prêtant une aventure amoureuse dont elle, Gertrude, a été privée vingt ans plus tôt. Geneviève n'est qu'un pion dans la vie intérieure de sa mère qui apparaît, moralement, comme une sorte de vampire qui cherche a faire sien le destin de sa fille. Et lorsque cette dernière revient à la maison, comme l'a prédit Henri, Gertrude trouve cette présence, cette expression de l'Autre, cette différence absolument intolérable. En existant d'une manière autonome, sa fille lui résiste, l'empêche de réaliser ses chimères.

Nous sommes donc en présence d'une mère dominatrice. Ses ennuis conjugaux, sa régression pitoyable ne doivent pas nous faire oublier qu'elle manipule ses enfants sans vergogne, dissimulant derrière ses crises de nerfs une constante volonté de puissance.

Cet instinct dominateur ressort également de ses rapports avec son fils Renaud, dit Boubou. Leur relation incestueuse apparaît d'abord sous un jour classique, Gertrude voulant liquider Henri pour se retrouver seule avec son fils: "Attends, Boubou! Reste, ton père va s'en aller." 23 On apprend aussi que Gertrude a voulu aller vivre à Paris avec Renaud ${ }^{24}$ et que, faute de réaliser ce projet, elle a sacralisé, en son absence, la chambre de son fils dont Geneviève nous apprend qu'il était défendu «d'y changer quoi que ce soit! C'était devenu un lieu de pélerinage ! 25

Par ailleurs, Renaud de retour, Gertrude le désire sexuellement. Alors qu'il sort de la douche, elle "s'appuie contre lui affectueusement ${ }^{26}$ en s'écriant : «C'est du béton armé ces cuisses-là ! ${ }^{26}$

Pourtant, c'est moins l'adulte que l'enfant, chez Renaud, qui est convoité par sa mère. Sa passion incestueuse est de nature régressive. En effet, elle désire moins son fils comme amant qu'elle ne cherche passionnément à retrouver en lui l'enfant. Elle veut lui sécher les cheveux ${ }^{27}$, lui faire ouvrir la bouche pour voir ses dents ${ }^{28}$, elle veut en fait le maîtriser. Ce qu'elle déplore, c'est qu'il soit devenu autonome, libre de ses jugements. Elle lui reproche son évolution, sa maturité : "Mais tu as changé! Tu as changé !"29, lui crie-t-elle avec dépit.

Il est également intéressant d'observer l'attitude de Renaud face à l'enveloppement maternel.

D'abord, il se sent coupable. II est mal à l'aise, vaguement honteux, de voir Gertrude "presque obscène à force d'abandon." ${ }^{0}$ Puis, il se montre suffisant, d'une certaine façon paternel, vis-à-vis sa mère. II déclame diverses sottises, par exemple: «Je ne renonce pas à l'idée de te changer (...) Je suis le seul qu'elle écoutera (...) A ton âge, maman !... " ${ }^{31}$ Enfin, il devient cruel, n'hésitant pas à assimiler, d'ailleurs à juste titre, sa mère à 
son mari : «Vous êtes pareils tous les deux (...) Desséchés! Complètement desséchés!»23

De son côté, Henri s'applique à éloigner Renaud de Gertrude ${ }^{32}$ comme il s'efforce, on l'a vu, de faire prendre conscience à sa femme qu'elle n'est pas sa fille. En plus de jouer le rôle du mari indigne, il est ainsi un facteur de vérité puisqu'il s'oppose aux fantasmes envahissants de Gertrude.

D'ailleurs, cette dernière renonce assez facilement à Renaud qui n'est pour elle qu'une occasion supplémentaire d'exprimer son désarroi profond. Elle déclare en effet à son fils qu'elle n'a aucune identité : «Est-ce que j'ai des idées? Est-ce que j'ai déjà eu une seule idée à moi dans toute ma vie? D'abord j'ai eu celle de ma famille... Puis celles de ton père... Et maintenant j'ai les tiennes! ${ }^{33} \mathrm{Si}$ l'on est tenté d'apporter une explication politique à Encore 5 minutes, c'est ici qu'il convient de le faire en observant que jamais Gertrude n'a pu s'affirmer comme personne, qu'elle a toujours été à la remorque de son entourage et que c'est là le destin d'une femme qui ne travaille pas, qui n'a pas un accès direct et personnel à l'argent. L'oisiveté, la dépendance économique mènent souvent à l'infantilisation et à la névrose.

Mais cette interprétation n'est pas explicite dans le texte de Loranger. D'un point de vue littéraire, ce n'est évidemment pas un tort. Après tout pour prendre un exemple illustre, Balzac n'écrit nulle part que son cuvre annonce la montée du capitalisme sauvage et le déclin de l'aristocratie terrienne. Au contraire même: dans sa naïveté, l'auteur de la Comédie humaine croyait servir l'Église et la monarchie.

C'est donc en inventant des personnages, en composant des métaphores, en nous racontant un cas particulier, bref c'est en faisant de la littérature que Françoise Loranger nous donne à voir une pièce susceptible mais seulement si le public le désire - de provoquer une réflexion féministe.

Il en va différemment des Fées ont soif dont l'intention est politique. L'intrigue y est agencée de manière à faire ressortir non seulement l'injustice faite aux femmes, mais aussi la quasi impossibilité de vivre la condition féminine. Ainsi, la statue, qui n'a pas d'enfant, se plaint: "Tous mes enfants m'ont été arrachés. " ${ }^{34}$ De même, Madeleine : "J'en aurais-tu voulu un p'tit bébé (...) de mon beau gars "35. Cependant, Marie, qui est mère de famille, se plaint également : ‘J'me sens niaiseuse. Inutile (...) C'te bébé-là, même quand i dort i m'enlève tout mon jus." 35 La boucle est bouclée. Dans les Fées ont soif, être ou ne pas être mère a la même fonction: permettre à l'auteur d'exprimer son insatisfaction. Mais cela ne donne pas une analyse approfondie du thème de la maternité.

Avec Denise Boucher, nous sommes trop souvent en présence d'un féminisme d'humeur, qui oscille entre le dépit individuel, insuffisamment élaboré et l'excès démagogique. Quand Marie affirme, par exemple: “Maman, dis-moi quelle bataille nous avons perdue un jour pour aboutir à être moins qu'un tapis ? "36, elle exagère et bien peu de féministes, hom-' 
mes et femmes, se reconnaîtront dans l'exhibition d'un tel complexe d'infériorité. De même, quand l'auteur fait dire à ses trois personnages: «Nous sommes des prisonnières politiques" ${ }^{37}$, elle ne rend nullement compte de la condition féminine québécoise. A force de dramatiser excessivement, on finit par écrire pour ne rien dire.

Tout compte fait, malgré son statut de bourgeoise et en dépit de l'absence de connotation féministe, la mère, chez Françoise Loranger, nous fait davantage réfléchir sur la maternité que les incantations de Denise Boucher.

\section{La sexualité}

Dans Encore 5 minutes, le thème sexuel le plus élaboré, c'est l'inceste. Nous avons déjà vu comment Gertrude désire Renaud et, plus bas, nous verrons comment la mère et le fils refoulent leur relation incestueuse. Dans les Fées ont soif, la sexualité est représentée d'une manière plus militante. II y est question de frigidité, de femme battue et de femme violée.

Marie et Madeleine se plaignent également de ne pas jouir. Cette dernière dit, en parlant de ses relations sexuelles avec ses clients : "J'ai introjecté (...) leurs désirs sans jamais les réaliser (...) J'ai sombré dans leurs folies sans jamais trouver les miennes (...) Ça fait si longtemps que je m'attends. " ${ }^{38}$ Ici Denise Boucher me semble montrer une certaine candeur. L'ambition d'une prostituee, ce n'est pas de jouir, mais de gagner de l'argent. Madeleine est une victime certes, mais ce n'est pas en exposant ses frustrations personnelles - au demeurant intéressantes - qu'on va approfondir le phénomène de la prostitution qui, en définitive, n'est pas traité dans les Fées ont soif.

De son côté, Marie affirme: "J'ai eu deux enfants et c'est comme si ma chair n'avait jamais été traversée. Pourquoi une mère n'aurait-elle pas joui ? "39 En effet, pourquoi pas? Encore que cette question ne soit pas spécifiquement féminine. Les hommes jouissent-ils nécessairement lorsqu'ils font des enfants? Néanmoins, l'auteur soulève ici une question importante en suggérant que c'est le modèle de la Vierge, omniprésent dans l'univers de Marie, qui explique sa frigidité : «Pauvre petite fille, tu as peutêtre joui. Peut-être que tu l'as oublié. Dans les hôpitaux psychiatriques, il y a plein de femmes qui se prennent pour moi. "40 En dénonçant le modèle virginal, et à travers lui toute forme de puritanisme, toute sexualité réglementée, les Fées ont soif manifestent un libéralisme indéniable.

La scène de la femme battue est également l'une des plus réussies de la pièce. Elle fait bien ressortir la détresse psychologique de Marie, qui est la condition de sa soumission. Dans la chanson au Père Noël ${ }^{41}$, qui ne lui apporte jamais de cadeau, qui ne la comprend pas, qui la laisse geler dehors, Marie exprime son besoin du Père. Et comme ce besoin est resté inassouvi, elle est une proie facile pour son mari qui la bat impunément. Après avoir été rouée de coups, insultée, humiliée, la pauvre femme est 
même persuadée qu'elle est coupable et qu'il lui appartient de s'amender : «Peut-être que je n'ai pas le tour avec lui (...) Ça doit être de ma faute si je l'agace autant. Faudrait que je fasse attention. "42 Et, d'une manière classique et navrante, elle s'imagine que la venue d'un enfant pourrait sauver son ménage : “ll voudrait tellement avoir d'autres enfants (...) ça nous raccorderait. » 42

Dans ce passage, Marie apparaît tellement brisée, tellement démunie que sa liberation, qui survient quelques pages plus loin, nous semble invraisemblable. Nous reviendrons plus bas sur cette question.

De la scène du viol, il y a peu à dire sinon qu'on ne peut que souscrire à ses conclusions.

D'abord, le violeur, qui peut être n'importe qui, s'efforce de rendre sa victime complice de son projet: "Les cochonnes comme toé, je les connais. " 43 Ensuite, trop souvent, il echappe a toute sanction : "ll y a eu la fin du procès. Le violeur fut innocenté. " 44

Le mérite des Fées ont soif, c'est de poser en termes clairs des questions graves, tels le viol et les multiples formes de violence faites aux femmes.

\section{Idéalisme et refoulement}

Quelle que soit son importance, tout groupe humain génère des idéaux qui sont proposés comme des normes à respecter. Ainsi, la société sexiste vise à imposer aux femmes des comportements stéréotypés, propres à les réduire aux rôles qu'on leur assigne. $A$ ce titre, les notions de Mère, d'Épouse, de Vierge ou de Putain apparaissent comme des valeurs d'enfermement. Ce sont des concepts réducteurs qui servent à contenir les femmes dans des conduites prévisibles. En cela, ces concepts sont des instruments de pouvoir.

Denise Boucher fait la critique de ces modèles qu'elle perçoit comme des carcans aliénants, propres à décerveler celles qui s'y soumettent.

Ainsi, dans la chanson du conditionnel ${ }^{45}$, les notions de beaute, de bonté, d'éternelle jeunesse, le mythe de la blondeur, le rôle de Mère, l'exaltation de la virginité sont successivement dénoncés. L'auteur réclame que la statue cesse d'être une sainte et que Marie et Madeleine ne soient pas considérées comme folles et hystériques parce qu'elles veulent quitter leur condition respective.

Mais il n'est pas facile d'échapper aux valeurs du milieu et nos trois femmes, en quête d'autonomie, sont progressivement terrorisées à l'idée de ne plus correspondre à l'attente ambiante. Avoir raison seul n'est jamais aisé et c'est pourquoi Marie et Madeleine décident de s'unir. Leur rencontre leur permet de confronter leurs difficultés respectives et de constater 
qu'elles ont des problèmes identiques. Elles s'aperçoivent qu'en n'etant pas conformes aux modèles ambiants, elles ne sont pas pour autant solitaires, isolées, exceptionnelles... La découverte de l'autre leur permet plus facilement d'échapper à tout complexe de culpabilité face aux pressions sociales.

Par exemple, Marie et Madeleine ont des règles difficiles. "Moé dans c'temps-là, chus gonflée, constipée, déprimée" 46 , nous dit l'une. "C'est pareil ajoute l'autre. J'me sens lourde. Pis, y m'sort toujours un gros bouton dans l'visage, juste ici. " ${ }^{46}$ Cela, c'est la réalité, partagée par les deux femmes. La norme, le mensonge idéaliste, le ronron publicitaire, c'est la statue qui le dénonce, avec une admirable vigueur : "Ces jours-là, madame, grâce à tantax, sentez-vous libre (...) tantax vous protège (...) Soyez moderne. Soyez libre. Soyez tantax. " ${ }^{46}$ Nous avons ici un exemple de ce que le thêâtre engagé peut donner de mieux : une description attentive du réel doublée d'une dénonciation vigoureuse du mensonge.

Dans les Fées ont soif, l'idéalisme s'oppose à la vérité de la vie. D'une part, il y a le monde; d'autre part, le discours sur le monde. C'est ce discours, en l'occurrence sexiste, que l'auteur dénonce. Sa démarche, en ce qu'elle vise à clarifier la condition féminine, à la décaper des couches de mensonges qui l'aseptisent, est essentiellement politique. Bien sûr, Denise Boucher apporte, à son tour, un discours sur les femmes que l'on peut trouver insuffisant, excessif, quelquefois même incohérent. Mais cela est secondaire. Ce qui compte, c'est qu'il y ait prise de parole, prise de conscience de manière à ce que les clichés séculaires et opportunistes qui entourent la réalité féminine soient ébranlés, dans le meilleur intérêt de tous.

Toujours synonyme de mensonge, l'idéalisme, dans Encore 5 minutes, apparaît comme une ruse de langage, comme un moyen employé par les personnages pour refouler des réalités désagréables.

Ce n'est plus d'un idéalisme conquérant dont il s'agit, comme celui de la société sexiste, mais d'un idéalisme pragmatique, intimiste, le plus souvent défensif. Chez Françoise Loranger, on vise autant à tromper autrui qu'à se donner une image tolérable de soi-même. Par exemple, Henri feint que c'est parce qu'il est un homme bien élevé qu'il ne gifle pas sa femme alors que c'est par impuissance, comme elle se plaît à le souligner : "Mais vas-y! Fais-le! Mais fais-le donc! (...) Hé ! non, tu ne peux pas ! ${ }^{47}$

C'est cependant dans la relation entre Gertrude et Renaud que l'auteur utilise le plus systématiquement des thèmes idéalistes, qui composent une sorte de langage indirect grâce auquel la mère et le fils peuvent s'aimer et se haïr sans se le dire ouvertement. Ils illustrent ainsi une des fonctions de la parole qui consiste à exprimer un référent refoulé grâce à un langage plus ou moins codé et en apparence rationnel. 
Par exemple, afin de se rapprocher de son fils, Gertrude prétend aimer les vieux meubles canadiens ${ }^{48}$ ainsi que la peinture moderne. ${ }^{48}$ Elle va même, d'une façon démagogique et régressive, jusqu'à faire l'éloge des jeunes chez qui elle trouve "une sorte de pureté" 49 tellement éclatante qu'elle estime que son mari et elles ne sont que "des mollusques comparés à eux " 49 .

On voit combien les goûts et les valeurs (l'artisanat, la peinture, etc.) ne sont ni innocents ni objectifs : ils ont une fonction chez ceux qui les professent. Ainsi, lorsque Renaud résiste à sa mère en ne répondant pas à ses avances, celle-ci utilise la norme grammaticale pour le dominer en lui expliquant qu'il faut dire corrigible et non « corrigeable» 50 .

Mais donner des leçons de français ne suffit pas à Gertrude. Renaud l'irrite tellement qu'elle désire le liquider. A cette fin, elle invente une fable dont la seule fonction est d'exprimer sa pulsion homicide. Elle feint d'apercevoir une dent bleue dans la bouche de son fils. Or, elle ajoute qu'elle a eu un cousin qui est mort justement de posséder une telle dent. II importe peu que cette anecdote soit fantaisiste, ce qui compte, c'est ce qu'elle suggère. Ici, le véritable référent du texte, c'est le trouble intérieur de Gertrude et non l'invraisemblable dent bleue de son lointain cousin Philippe.

De son côté, Renaud utilise également le langage indirect. II s'habille mal et parle volontairement d'une manière vulgaire à seule fin d'irriter son père. Celui-ci commet d'ailleurs l'erreur de réagir à cette provocation. II s'indigne, procurant ainsi un réel plaisir à Renaud.

Ce dernier souhaite par ailleurs introduire son amie Corinne chez lui. II veut justement l'installer dans la chambre de sa sœur, que sa mère s'efforce désespérément d'occuper.

Il est intéressant d'observer combien les motivations de chacun au sujet de Corinne restent dissimulées.

Par exemple, Renaud prétend recevoir Corinne à la maison parce qu'elle est pauvre et méritante. Et quand sa mère refuse, tout en offrant de lui louer une chambre près de l'université, il s'y oppose parce que "ce serait lui faire la charité et qu'elle ne demande pas la charité „51. II propose alors de faire habiter Corinne dans une de leurs chambres de bonne. Mais cela "serait humiliant pour elle»52, riposte Gertrude.

Chacun reste sur sa position. Renaud maintient son désir; Gertrude, sa résistance.

A bout d'arguments précis, chacun se lance dans une diatribe idéologique. Déjà de gauche, le bon Renaud s'enflamme sur le "sort d'un être humain " 53 et s'inquiète des "petites préoccupations personnelles et bourgeoises" 53 de sa mère. Nettement à droite, celle-ci fait une violente sortie contre les "maudits pauvres " ${ }^{51}$ qui veulent sa maison, voire son lit.

Finalement, la situation se clarifie. On sort de cette glu idéaliste, de ce bavardage idéologique, moral, essentiellement opportuniste quand Renaud 
manifeste enfin son pouvoir sur sa mère en lui disant: «Ou bien Corinne vient passer ici le temps qu'il lui faut pour écrire sa thèse, ou bien c'est moi qui m'en vais. "54 Désiré par sa mère, Renaud a les moyens de la briser. $A$ ce titre, comme souvent les œuvres qui traitent de l'œdipisme, Encore 5 minutes est une fable politique. Ici, c'est le fils qui triomphe. Ailleurs, par exemple dans les romans de Jacques Godbout, c'est la femme, c'est-àdire la mère, qui l'emporte.

Examinons maintenant les dénouements des deux pièces étudiées. Dans chaque cas, les auteurs prétendent libérer leurs héroïnes. Qu'en est-il exactement?

\section{Une douteuse libération}

En critiquant la condition féminine telle que vécue dans notre société, les Fées ont soif et Encore 5 minutes visent, plus ou moins consciemment selon les cas, à libérer la femme. La critique de Denise Boucher est systématique. Elle dénonce des comportements et un discours dont souffrent toutes les femmes. Ses personnages sont des archétypes qui servent à véhiculer une idéologie superficiellement contestataire, mais en fait conforme à la mode régnante. À l'encan des idées, le féminisme n'est-il pas ce qui se vend le mieux actuellement? C'est donc moins l'intention progressiste de l'auteur qu'il convient d'examiner que son projet exact de libération des femmes, tel qu'il se dégage de sa pièce.

D'abord, Denise Boucher prône l'union. Marie et Madeleine doivent se rencontrer, confronter leurs difficultés et se découvrir mutuellement. Ensuite, avec la statue, elles fredonnent la «chanson d'errance» dans laquelle elles en appellent à toutes les femmes et à quelques hommes pour former un front de libération dont le programme me semble incontestable dans sa générosité, juste dans ses principales dénonciations et peu convaincant dans les valeurs qu'il tente de substituer à l'ordre qu'il attaque.

Pour l'épouse, il s'agit de quitter son mari, un homme ignoble, stupide et cruel. II n'est nullement nécessaire d'être féministe pour souhaiter cette séparation. Et les difficultés qu'éprouve Marie à se retrouver seule ne relèvent pas d'une analyse politique. Homme ou femme, quiconque opère un changement radical dans sa vie risque d'éprouver un sentiment désagréable, plus ou moins grave selon sa sensibilité.

Quant à Madeleine, son ambition est également inattaquable : elle veut cesser de se prostituer. Elle sent un besoin de liberté qu'elle assimile à "un grand vent nécessaire 55 . L'image est vague et dissimule un projet éculé, qui traîne depuis longtemps dans la littérature consacrée à la prostitution. Madeleine désire en effet s'ouvrir « un p'tit commerce. Un p'tit magasin de coupons. 56 Désormais, elle vendra de "la soie (...) du velours (...) du beau coton. "56 Du trottoir au comptoir : cela fait un gentil mélo, mais on l'a trop souvent entendu pour s'y intéresser de nouveau. 
Denise Boucher est plus heureuse lorsqu'elle nous parle de la mère de Madeleine, qui, épuisée par sa pauvreté, ses neuf enfants et sa fatigue, "sortait (...) ramassait une brique (...) pétait une vitrine. " 57 Cet acte de révolte était immédiatement assimilé à un acte de folie. Conduite à l'asile, la pauvre femme était baignée, traitée, gavée de pilules. Au bout de quelques jours, on la libérait pourvu que son mari vienne “signer pour la sortir. „58 En apparence sans valeur politique, le comportement anti-social de cette femme trouve sa véritable signification grâce à l'éclairage féministe auquel il est soumis. Michel Foucault a bien montré combien l'asile et la prison exprimant violemment, d'une manière à la fois concrète et métaphorique, les tensions sociales, les luttes de pouvoir qui définissent nos sociétés.

Par ailleurs, ce n'est pas en féminisant les mots soleil, ciel ou œuf que l'auteur va faire avancer la cause des femmes ${ }^{59}$. II y a des cas où le ridicule dessert les causes les plus justes.

Comme souvent les œuvres engagées, Les Fées ont soif sont plus efficaces pour dénoncer l'injustice que pour découvrir des idées nouvelles, vraiment mobilisatrices. II ne suffit pas de murmurer en prenant un air navré et méchant: "Cellule Famille Foyer Religion" 60 pour convaincre. De même, dans les dernières pages de l'œuvre, les personnages nous répètent à l'envi d'imaginer l'amour et le bonheur. Ce vœu pieux, exprimé dans un style assez pauvrement poétique, me semble une conclusion bien faible pour une pièce dont l'intérêt réside avant tout dans la critique précise de certaines injustices faites aux femmes.

Sans suivre complètement Jean-Cléo Godin qui parle de «détermination lucide (...) inébranlable "61 au sujet du départ de Gertrude à la fin d'Encore 5 minutes, on peut relever divers éléments positifs dans le dénouement de cette pièce.

Il y a d'abord la lucidité de l'héroïne qui demande : «Quelle sorte de poison suis-je donc pour qu'on soit obligé, les uns après les autres, de vous détacher de moi?" 62 Gertrude prend ici conscience de son comportement possessif, de l'inadmissible pression affective qu'elle impose aux siens.

Cela la conduit, en quittant la maison, à offrir sa chambre à Corinne, c'est-à-dire à rendre son fils à sa vie amoureuse.

De même, en s'éloignant, Gertrude atteint enfin son mari qui est ulcéré par ce soudain désir d'indépendance. II s'efforce de minimiser l'événement en feignant de croire qu'elle veut seulement partir en voyage. II finit même, devant la fermeté de sa femme, par exhiber lâchement son pouvoir économique. II lui dit : «De quoi vivras-tu? (...) L'argent, c'est moi ! 63 .

Comment croire cependant à sa volonté de libération? Elle a montré un tel état d'asservissement qu'on imagine mal qu'elle puisse tout à coup 
trouver en elle-même les moyens de s'assumer. Mais cela, bien sûr, est un faux problème. Après tout, Gertrude n'est qu'un personnage dramatique à travers lequel l'auteur exprime une double réalité : d'abord, l'aliénation d'une bourgeoise aux prises avec un grave problème d'identité; ensuite, la nécessité de surmonter cette aliénation. Peu vraisemblable d'un point de vue psychologique, le dénouement d'Encore 5 minutes est par ailleurs nécessaire d'un point de vue féministe. À ce titre, on peut rapprocher le personnage de Gertrude de celui de Marie dans les Fées ont soif.

Les conclusions des deux pièces restent néanmoins un peu faibles. Pour l'amateur de théâtre, les héroïnes de Denise Boucher et Françoise Loranger sont plus convaincantes aliénées qui libérées. Cela ne surprend guère. On sait depuis longtemps que les personnages littéraires sont captivants dans la mesure où ils ont des obstacles à surmonter. Triomphantes et libres, Marie, Madeleine la Statue et Gertrude perdent vite leur intérêt dramatique. II est temps que le rideau tombe.

1. Jean-Pierre Crête, Françoise Loranger, Montréal, Ed. Leméac, 1974, p. 101.

2. Denise Boucher, Les Fées ont soif, Montréal, Ed. Intermède, novembre 1978, p. 83 à 87 . Intermede a réédité la pièce en septembre 1979. Cette seconde édition comporte des variantes, parfois substantielles. Dans ce travail, nous ferons état de ces modifications.

3. Jean-René, Ethier, "Fées ou Sorcières", Relations, janvier 1979, p. 27.

4. Denise Boucher, op. cit., p. 97.

5. Ibid., p. 83 .

6. Ibid., p. 88.

7. Ibid., p. 94.

8. Ibid., p. 90 .

9. Ibid., p. 91.

10. Ibid., p. 92-93.

11. Françoise Loranger, Encore 5 minutes, Montreal, Ed. le Cercle du Livre de France, octobre 1971, p. 27.

12. Ibid., p. 26.

13. Ibid., p. 7.

14. Ibid., p. 52.

15. Ibid., p. 7, 10, 24, 25.

16. Ibid., p. 66.

17. Ibid., p. 39.

18. Ibid., p. 22.

19. Ibid., p. 25.

20. Ibid., p. 31.

21. Ibid., p. 57.

22. Ibid., p. 58.

23. Ibid., p. 23.

24. Ibid., p. 19.

25. Ibid., p. 64.

26. Ibid., p. 11.

27. Ibid., p. 8.

28. Ibid., p. 16.

29. Ibid., p. 20.

30. Ibid., p. 44.

31. Ibid., p. $72,74,78$. 
32. Ibid., p. 32 à 36.

33. Ibid., p. 45.

34. Denise Boucher, op. cit., p. 118.

35. Ibid., p. 117.

36. Ibid., p. 120.

37. Ibid., p. 121-122.

38. Ibid., p. 99.

39. Ibid., p. 97.

40. Ibid., p. 98.

41. Ibid., p. 108

42. Ibid., p. 114.

43. Ibid., p. 135.

44. Ibid., p. 142.

45. Ibid., p. 101.

46. Ibid., p. 95.

47. Françoise Loranger, op. cit., p. 40-41.

48. Ibid., p. 11-12.

49. Ibid., p. 37.

50. Ibid., p. 18.

51. Ibid., p. 47.

52. Ibid., p. 46.

53. Ibid., p. 12-13.

54. Ibid., p. 50.

55. Denise Boucher, op. cit., p. 133.

56. Ibid., p. 132.

57. Ibid., p. 128. Dans la seconde édition, cette scène disparaît. Pour obtenir une plus grande efficacité dramatique? En tout cas, d'un point de vue féministe, cette suppression a pour effet d'abolir la solidarité qui unissait les mères et les filles dans leur combat contre la société masculine.

58. Ibid., p. 129.

59. Ibid., p. 83, 126.

60. Ibid., p. 121.

61. Jean-Cléo Godin et Laurent Mailhot, Le Théatre québécois, Montréal, Ed. Hurtubise, 1970, p. 115.

62. Françoise Loranger, op. cit., p. 72.

63. Ibid., p. 79. 